\title{
PREDICCIÓN DE LA INTELIGENCIA EMOCIONAL A TRAVÉS DE LA SATISFACCIÓN DE LAS NECESIDADES PSICOLÓGICAS BÁSICAS EN LAS CLASES DE EDUCACIÓN FÍSICA
}

\author{
Manuel Gómez-López \\ Departamento de Actividad Física y Deporte, Facultad de Ciencias del Deporte \\ Universidad de Murcia, España \\ mgomezlop@um.es \\ Antonio Granero-Gallegos \\ Departamento de Educación, Facultad de Ciencias de la Educación \\ Universidad de Almería, España \\ agranero@ual.es
}

Recepción Artículo: 22 enero 2020

Admisión Evaluación: 4 marzo 2020

Informe Evaluador 1: 1 abril2020

Informe Evaluador 2: 7 abril 2020

Aprobación Publicación: 20 abril 2020

\section{RESUMEN}

Los objetivos de este trabajo fueron: describir el nivel de satisfacción de las necesidades psicológicas básicas y de inteligencia emocional de estudiantes adolescentes de secundaria, y comprobar la capacidad de predicción de la primera en la segunda. Para ello se respondieron a las versiones validadas al castellano de la Basic Psychological Needs in Exercise Scale y la Trait Meta-Mood Scale. La muestra estuvo compuesta por 464 alumnos de secundaria de 12 a 17 años. Los resultados del análisis descriptivo y de correlación indicaron que la necesidad psicológica que más sienten tener satisfecha fue la relación con los demás y que fue la dimensión reparación emocional la que obtuvo mayor puntuación. Por otro lado, mediante análisis de regresión lineal se halló que la necesidad psicológica que mayor predicción tuvo sobre las necesidades psicológicas básicas fue la relación con los demás. Los resultados obtenidos indicaron que las personas con mayor necesidad de relacionarse tenderán a desarrollar una mejor inteligencia emocional.

Palabras clave: autonomía; competencia; emociones; adolescencia; educación física

\section{ABSTRACT}

Predicting emotional intelligence by meeting basic psychological needs in physical education classes. The aims of this study were: to describe the level of satisfaction of the basic psychological and emotional intelligence needs of adolescent high school students, and to verify the predictive capacity of the first over the second. For this purpose, the validated Spanish versions of the Basic Psychological Needs in Exercise Scale and the Trait Meta-Mood Scale were used. The sample was composed of 464 secondary school students from 12 to 17 years old. The results of the descriptive and correlation analysis indicated that the psychological 
need they felt most satisfied was their relationship with others and that it was the emotional repair dimension that scored highest. On the other hand, through linear regression analysis it was found that the psychological need that was most predictive of basic psychological needs was the relationship with others. The results obtained indicated that people with greater need to relate will tend to develop better emotional intelligence.

Keywords: autonomy; competence; emotions; adolescence; physical education

\section{ANTECEDENTES DE LA TEMÁTICA A TRATAR}

La literatura ha demostrado que el bienestar psicológico de los practicantes de actividad física se relaciona positivamente con la motivación autodeterminada (González-Serrano, Huéscar, \& Moreno-Murcia, 2013; Reis, Sheldon, Gable, Roscoe, \& Ryan, 2000).

La teoría más utilizada para explicar la motivación de las personas tanto en el contexto deportivo como en el educativo, concretamente en las clases de Educación Física (EF en adelante) ha sido la teoría de la autodeterminación (TAD) (Deci \& Ryan, 1985, 1991, 2000; Ryan \& Deci, 2000, 2017), la cual diferencia el grado en el que las personas realizan sus acciones de forma voluntaria. Dentro de este postulado, una de las miniteorías que ha tenido mayor relevancia en el contexto educativo es la teoría de las necesidades psicológicas básicas (e.g., Baena-Extremera, Gómez-López, Granero-Gallegos, \& Martínez-Molina, 2016; Barkoukis, Hagger, Lambropoulos, \& Tsorbatzoudis, 2010; Martínez Rubio, Granero-Gallegos, \& Gómez-López, 2020; Méndez Giménez, Fernández Río, \& Cecchini Estrada, 2013). Esta teoría se fundamenta en que todas las personas tenemos unas necesidades psicológicas (autonomía, competencia y relación con los demás) (NPB en adelante) que son innatas y universales y cuya satisfacción es esencial para el crecimiento psicológico, integridad y bienestar de la persona (Deci \& Ryan, 1985, 2000; Ryan \& Deci, 2000).

Para Deci y Ryan (1991) la necesidad de autonomía se caracteriza por los esfuerzos de las personas por sentirse ellas mismas el origen de las acciones que realizan y el poder determinar su propio comportamiento y aprendizaje autónomo. Los resultados del estudio realizado por Baena-Extremera et al. (2016) demostraron la importancia de la autonomía, reflejando que los estudiantes que participaron en la toma de decisiones dentro de las clases de EF, se sintieron más eficaces y percibieron una buena relación con sus compañeros, alcanzando de este modo una motivación más autodeterminada, la cual está caracterizada por la diversión, satisfacción y la valoración positiva de la actividad desarrollada en clase (Moreno, Hernández, \& González-Cutre, 2009; Moreno-Murcia, González-Cutre, Chillón, \& Parra, 2008). El estudio realizado por Reeve, Jang, Carrell, Barch y Jeon (2004) con niños aportó que cuando estos niños percibieron un soporte de autonomía por parte del docente, sintieron mayor satisfacción con la vida. Por lo tanto cuando el docente es capaz de crear un clima de apoyo a la autonomía en clase, los estudiantes se sienten escuchados, importantes, más comprendidos y por lo tanto experimentan una sensación de libertad para actuar (Baena-Extremera et al. 2016).

La segunda necesidad es la competencia, la cual busca ser capaz de regular o controlar el resultado y sentir eficacia en las acciones realizadas. Según Deci y Ryan (2000) la competencia y la autonomía tienen mayor influencia en la motivación autodeterminada, que la relación con los demás. Es más, diferentes estudios reflejaron los efectos beneficiosos que la necesidad de competencia y de autonomía tienen en la autoestima y la satisfacción con la vida, a través de la motivación autodeterminada (Balaguer, Castillo, \& Duda, 2008; Moreno \& Vera, 2011).

Finalmente, la tercera necesidad es la relación con los demás, que hace alusión a los esfuerzos que realizan las personas para relacionarse con los demás, y se caracteriza por el sentimiento de aceptación con el grupo. Es decir, el alumno que cree que es valorado y respetado dentro del grupo de iguales tiene sentimientos afectivos relacionados con el rendimiento en clase y la autoestima (Duncan, 1993), por lo que, la opinión positiva del grupo favorece la confianza en sí mismo y el desarrollo de su autoestima (Moreno \& Vera 2011). Además, se ha demostrado que cuando los estudiantes perciben que son aceptados por los demás, y tienen apoyo del grupo de iguales muestran un mayor bienestar subjetivo (Cava \& Musitu, 2000). Cuando el docente enfatiza el rol de cada uno de los estudiantes satisface las NPB, en especial la relación con las demás (Méndez-Giménez et al., 2013). 
En las clases de EF, la motivación del alumnado tiene dependencia de la satisfacción de las NPB, siendo el docente el principal responsable de conseguir la satisfacción de las mismas (García-González, Aibar, Sevil, Almolda, \& Clemente, 2015; Ntoumanis, 2001; Ryan \& Deci, 2000). Cuanto más satisfechas tengan los alumnos estas necesidades, mayor será su motivación autodeterminada (Franco \& Coterón, 2017; McDonough \& Crocker, 2007). Por otro lado, diferentes estudios realizados tanto en el entorno educativo (Cera, Almagro, Conde, \& Sáenz-López, 2015; Fierro-Suero, Almagro, \& Sáenz-López, 2019) como en el deportivo (Martín \& Guzmán, 2012; Watson \& Kleinert, 2018) hallaron correlaciones positivas entre la satisfacción de las NPB y la inteligencia emocional (IE en adelante), es más, Fierro-Suero et al. (2019), demostraron que estas NPB fueron predictoras de la IE.

Por IE entendemos la habilidad para percibir, asimilar, comprender y regular las propias emociones y la de Ios demás, promoviendo un crecimiento emocional e intelectual (Mayer \& Salovey, 1990, 1997). De este modo, las personas emocionalmente inteligentes son capaces por un lado de atender a las emociones percibidas en su entorno más próximo, comprendiendo sus posibles causas y consecuencias y por otro de desarrollar estrategias para regular los estados emocionales (Mayer \& Salovey, 1997; Mayer, Salovey, \& Caruso, 2000; Pena \& Extremera, 2012). De ahí, que el desarrollo de la IE sea un importante indicador de éxito en diferentes aspectos de la vida (Bar-On, 2002; Saarni, 1999; Goleman, 1995).

\section{OBJETIVOS DE LA INVESTIGACIÓN}

En primer lugar, describir el nivel de satisfacción de las NPB y de IE de estudiantes adolescentes de secundaria y, en segundo lugar, comprobar la capacidad de predicción de la satisfacción de las NPB sobre la IE del alumnado.

\section{MUESTRA Y/O PARTICIPANTES}

Participaron 464 estudiantes de educación secundaria obligatoria (ESO) (238 chicos, 51.3\%; 226 chicas, 48.7\%) con edades comprendidas entre 12 y 17 años ( $M=14.08$; $D T=1.53)$, y pertenecientes a cuatro centros de carácter público de la Región de Murcia.

\section{METODOLOGÍA Y/O INSTRUMENTOS UTILIZADOS}

La muestra se seleccionó mediante un muestreo no probalístico intencional en base a un diseño descriptivo correlacional transversal (Montero \& León, 2007) y se utilizó un cuestionario con las siguientes escalas:

Necesidades Psicológicas Básicas. Se utilizó la versión española adaptada a la EF (BPN-PE; Menéndez \& Fernández-Río, 2018) de la escala original Basic Psychological Needs in Exercise Scale (BPNES; Vlachopoulos y Michailidou, 2006; Vlachopoulos, Katartzi, \& Kontou, 2011). La escala está compuesta por 12 ítems (cuatro por factor) que evalúan la autonomía (e.g. "Pienso que la forma en que se imparte la Educación Física es tal y como a mí me gusta"), competencia (e.g. "Creo que mejoro incluso en las tareas que la mayoría de los compañeros considera difíciles") y relación (e.g. "Las relaciones con mis compañeros de clase son muy amistosas"). Las respuestas se recogieron en una escala tipo Likert de cinco puntos que oscilaba desde totalmente en desacuerdo (1) a totalmente de acuerdo (5). En el presente estudio, el análisis de consistencia interna fue: autonomía, alfa de Cronbach $(\alpha)=.71$; competencia, $\alpha=.71$; relación con los demás, $\alpha=.82$.

Inteligencia emocional percibida. Se utilizó la versión adaptada al español (Fernández-Berrocal, Extremera, \& Ramos, 2004) de la escala original denominada Trait Meta-Mood Scale (TMMS; Salovey, Mayer, Goldman, \& Palfai, 1995). La escala mide el nivel de inteligencia emocional percibida a través de 24 ítems, distribuidos en tres subescalas de 8 ítems cada una, que evalúan la atención emocional, la claridad de sentimientos, y la reparación emocional. La subescala atención emocional expresa el grado en que las personas advierten y piensan acerca de sus sentimientos (e.g. "Presto mucha atención a los sentimientos"), la subescala claridad emocional evalúa la capacidad de entender el estado de ánimo de uno mismo (e.g. "Tengo claros mis sentimientos"), la subescala reparación emocional evalúa el grado en que los individuos moderan y regulan sus sentimientos (e.g. 


\section{PREDICCIÓN DE LA INTELIGENCIA EMOCIONAL A TRAVÉS DE LA SATISFACCIÓN DE LAS NECESIDADES PSICOLÓGICAS BÁSICAS EN LAS CLASES DE EDUCACIÓN FÍSICA}

"Cuando estoy triste, pienso en todos los placeres de la vida"). La escala utilizada estaba precedida por la frase introductoria: "A continuación encontrará algunas afirmaciones sobre sus emociones y sentimientos...". Las respuestas se recogieron en una escala tipo Likert de cinco puntos que oscilaba desde nada de acuerdo (1) a totalmente de acuerdo (5). En el presente estudio, el análisis de consistencia interna fue: atención emocional, $\alpha$ $=.87$; claridad de sentimientos, $\alpha=.86$; reparación emocional, $\alpha=.82$.

Para poder asistir a los centros educativos y realizar el trabajo de campo, se obtuvo autorización de la Dirección de cada Centro, Consejo Escolar y profesores de de la asignatura de EF de los cursos asignados en la toma de datos, y de los padres de los propios estudiantes mediante una carta en la que se explicaban los objetivos de la investigación y cómo se realizaría, acompañando un modelo del instrumento. Previamente a la pasación del instrumento, se informó a los estudiantes de la finalidad del estudio, de su voluntariedad, absoluta confidencialidad de las respuestas y manejo de los datos, que no había respuestas correctas o incorrectas y solicitándoles máxima sinceridad y honestidad. El instrumento fue autoadministrado con aplicación masiva en una jornada escolar y con consenso y adiestramiento previo de los encuestadores. Todo el trabajo de campo se llevó a cabo de manera anónima y siempre en presencia de dos encuestadores y del profesor de Educación Física. Para la cumplimentación del cuestionario se requirió una media de 20 minutos de la clase de EF, variando ligeramente en función de la edad. Todo el trabajo de campo se llevó a cabo siempre en presencia de dos encuestadores y del profesor de EF. Únicamente aquellos estudiantes que contaban con un consentimiento informado de los progenitores y/o tutores participaron en la investigación. Finalmente señalar que se obtuvo el informe positivo de la Comisión de Ética de Investigación de la Universidad de Murcia (ID: 1900/2018).

Para la consecución del objetivo planteado se llevó a cabo el siguiente análisis de datos. En primer lugar, fueron calculados los estadísticos descriptivos, correlaciones, consistencia interna de cada subescala (alfa de Cronbach), y los índices de asimetría y curtosis, oscilando los valores entre -1.26 y 1.31, lo que indica semejanza con la curva normal de forma univariada y permite la utilización de estadística paramétrica. Para comprobar la relación de predicción entre las subescalas del BPN-PE y las del TMMS-24 se llevó a cabo un análisis de regresión linear múltiple. El cálculo estadístico se realizó con el paquete SPSS en su v.24 para Windows.

\section{RESULTADOS ALCANZADOS}

Los estadísticos descriptivos de las diferentes subescalas se exponen en la Tabla 1. Respecto a las dimensiones de la satisfacción de las NPB, los valores medios más altos se encontraron en la relación con los demás, seguidos de la competencia y de la autonomía. Por otro lado, en las dimensiones de la IE, las puntuaciones son adecuadas y las más altas correspondieron a la reparación emocional, seguida de la atención emocional y de la claridad de sentimientos.

Respecto a las correlaciones, se observa que son altas, positivas y estadísticamente significativas $(p<.0001)$ entre las subescalas de las NPB. La correlación entre las dimensiones de la IE también son positivas y estadísticamente significativas $(p<.0001)$, destacando el valor alcanzado entre la claridad de sentimientos y la reparación emocional (.50).

Tabla 1. Estadísticos descriptivos y correlaciones entre las subescalas del BPN-PE y TMMS-24.

\begin{tabular}{|c|c|c|c|c|c|c|c|c|c|}
\hline & Subescalas & $\mathbf{M}$ & DT & $\mathbf{1}$ & 2 & 3 & 4 & 5 & 6 \\
\hline \multirow{3}{*}{$\begin{array}{l}1 . \\
2 . \\
3 .\end{array}$} & \multirow{3}{*}{$\begin{array}{l}\text { Autonomía } \\
\text { Competencia } \\
\text { Relación con los } \\
\text { demás }\end{array}$} & 3.13 & .82 & \multirow[t]{6}{*}{-} & $.51^{\text {*36 }}$ & $.38^{* 3 *}$ & $.10^{*}$ & $.24^{* * 3}$ & $.26^{* 3}$ \\
\hline & & 3.62 & .83 & & - & $.50^{* * *}$ & .07 & $.29^{* *}$ & $.31^{* * 4}$ \\
\hline & & 4.10 & .89 & & & - & $.13^{* * *}$ & $.26^{* * *}$ & $.32^{* *}$ \\
\hline 4. & Atención emocional & $\begin{array}{c}26.7 \\
2\end{array}$ & 7.18 & & & & - & $.26^{* *}$ & $.19^{* * *}$ \\
\hline 5. & $\begin{array}{l}\text { Claridad de } \\
\text { sentimientos }\end{array}$ & $\begin{array}{c}25.9 \\
4\end{array}$ & 6.90 & & & & & - & $.50^{* *}$ \\
\hline 6. & Reparación emocional & $\begin{array}{c}27.9 \\
2\end{array}$ & 6.72 & & & & & & - \\
\hline
\end{tabular}

Nota: * La correlación es significativa al .01; * La correlación es significativa al .05; $M=$ Media; $D T=$ Desviación típica. 
Con el objeto de comprobar en qué medida las diferentes dimensiones del BPN-PE predicen la IE percibida, se llevó a cabo un análisis de regresión lineal múltiple. En este análisis, Ios factores de la escala de NPB actuaron como variables predictoras y cada uno de los factores de la escala de IE como variable criterio 0 dependiente (Tabla 2). Inicialmente, se evaluó el índice de tolerancia e independencia de las variables incluidas en la ecuación de regresión. El índice de tolerancia alcanzó valores entre .81 y .98 y el factor de inflación de la varianza (VIF) presentó valores entre 1.00 y 1.47, por lo que estos datos indican que la probabilidad de error derivada de la posible colinealidad queda descartada (Hair, Black, Babin, \& Anderson, 2009). El estadístico Durbin-Watson estuvo comprendido entre 1.87 y 1.98, lo que permite afirmar la independencia de los datos (Gil, 2003).

En la Tabla 2 se exponen los resultados del análisis de regresión lineal. En relación con la atención emocional se alcanzó un $8 \%$ de la varianza total explicada; en este caso, la subescala de relación con los demás resultó estadísticamente significativa como predictora positiva ( $\beta=.11 ; p=.042$ ) de la atención emocional, de tal manera que una mejor relación con los compañeros supone mayor probabilidad de mejorar la atención emocional. Restecto a la claridad de sentimientos, ( $11 \%$ de varianza total explicada), la satisfacción de la competencia ( $B=$ $.17 ; p=.002)$ y la satisfacción de la relación con los demás ( $B=.14 ; p=.008)$ se muestran como predictores positivos y estadísticamente significativos. Finalmente, en la subescala de reparación emocional (14\% de varianza total explicada), la relación con los demás se mostró como predictor positivo estadísticamente significativo más fuerte $(B=.21 ; p<.0001)$, seguida de la competencia $(B=.15 ; p=.005)$.

Tabla 2. Regresión lineal múltiple. Correlaciones, pesos beta estandarizados (B) y varianza total explicada (R2) para cada paso. Variables dependientes: atención emocional, claridad de sentimientos y reparación emocional, respectivamente.

\begin{tabular}{|c|c|c|c|c|c|}
\hline Subescala & $F$ & $R^{2}$ & $\beta$ & $\mathbf{t}$ & $p$ \\
\hline $\begin{array}{l}\text { Atención emocional } \\
\text { Autonomía } \\
\text { Competencia } \\
\text { Relación con los } \\
\text { demás }\end{array}$ & 2.91 & .08 & $\begin{array}{c}.06 \\
-.02 \\
.11\end{array}$ & $\begin{array}{l}1.14 \\
-.27 \\
2.04\end{array}$ & $\begin{array}{l}.254 \\
.784 \\
.042\end{array}$ \\
\hline $\begin{array}{l}\text { Claridad de } \\
\text { sentimientos } \\
\text { Autonomía } \\
\text { Competencia } \\
\text { Relación con los } \\
\text { demás } \\
\end{array}$ & 18.78 & .11 & $\begin{array}{l}.10 \\
.17 \\
.14\end{array}$ & $\begin{array}{l}1.88 \\
3.13 \\
2.66\end{array}$ & $\begin{array}{l}.060 \\
.002 \\
.008\end{array}$ \\
\hline $\begin{array}{l}\text { Reparación } \\
\text { emocional } \\
\text { Autonomía } \\
\text { Competencia } \\
\text { Relación con los } \\
\text { demás } \\
\end{array}$ & 25.23 & .14 & $\begin{array}{l}.10 \\
.15 \\
.21\end{array}$ & $\begin{array}{l}1.96 \\
2.82 \\
4.17\end{array}$ & $\begin{array}{l}.050 \\
.005 \\
.000\end{array}$ \\
\hline
\end{tabular}

Nota: $\mathrm{R}^{2}=$ Varianza total explicada; $\beta=$ pesos beta estandarizados.

\section{DISCUSIÓN}

Los objetivos del estudio fueron, en primer lugar, describir el nivel de satisfacción de las NPB y de IE de estudiantes adolescentes de secundaria y en segundo lugar, comprobar la capacidad de predicción de la satisfacción de las NPB sobre la IE del alumnado.

Respondiendo al primer objetivo, los resultados mostraron que las NPB que más sienten tener satisfechas 


\section{PREDICCIÓN DE LA INTELIGENCIA EMOCIONAL A TRAVÉS DE LA SATISFACCIÓN DE LAS NECESIDADES PSICOLÓGICAS BÁSICAS EN LAS CLASES DE EDUCACIÓN FÍSICA}

fueron en primer lugar la relación con los demás, seguida de la competencia y por último la autonomía. Por lo que se refleja que la mayoría de los estudiantes se sienten aceptados por el grupo. En relación a la IE, Ios resultados aportaron puntuaciones adecuadas, siendo dimensión reparación emocional la más alta, seguida de la atención emocional y de la claridad de sentimientos. Estos resultados reflejaron que la mayoría de los estudiantes moderan y regulan adecuadamente sus sentimientos.

En relación al segundo objetivo, los resultados demostraron que la necesidad psicológica que mayor capacidad de predicción tuvo sobre las tres dimensiones de la IE fue la relación con los demás, especialmente en la dimensión atención emocional, ya que fue la única necesidad psicológica predictora. En cuanto a la dimensión claridad de sentimientos, está fue predicha sobre todo por la percepción de competencia. Y finalmente, la reparación emocional, fue predicha sobre todo por la relación con los demás, seguida de la percepción de competencia. Estos resultados son similares a los encontrados recientemente por Méndez-Giménez, Cecchini y GarcíaRomero (2018) y Callea, De Rosa, Ferri, Lipari y Costanzi (2019), puesto que se demostró una asociación positiva y significativa entre la necesidad de relación con los demás y las tres dimensiones emocionales que integran la IE. Recordar que según la Teoría de la Autodeterminación, las NPB contienen componentes tanto cognitivos como afectivos y la necesidad de relacionarse con los demás es un importante factor de motivación para el desarrollo de la IE (Deci \& Ryan, 2000). Por lo tanto, según los resultados anteriores, los estudiantes con mayor capacidad para comprender y controlar sus emociones y empatizar con sus compañeros, tendrán mayores probabilidades de satisfacer su relación con los demás. Del mismo modo, Fernández-Ozcorta, Almagro y Sáenz-López (2014) en el contexto universitario hallaron que la IE percibida predijo la satisfacción de las tres NPB. En cambio, los resultados coinciden parcialmente con los hallados anteriormente por Cera et al. (2015), en cuyo estudio se demostró una relación positiva y significativa entre las tres NPB y la atención emocional y entre la necesidad de relación con los demás y la dimensión reparación emocional.

Por otro lado, en el estudio realizado por Fierro-Suero et al. (2019), la IE estuvo compuesta por cinco dimensiones: inteligencia interpersonal, inteligencia intrapersonal, adaptabilidad emocional, manejo del estrés y estado de ánimo general. Los resultados mostraron correlaciones positivas entre las tres NPB y la IE intrapersonal, IE interpersonal, el estado de ánimo general, la adaptabilidad emocional y la IE general (puntuación del cuestionario al completo). Asimismo, se halló que la satisfacción de la necesidad de competencia en las clases de EF predijo positivamente la IE general.

Por lo tanto, los resultados obtenidos indicaron que las personas con mayor necesidad de relacionarse tenderán a desarrollar una mejor IE. A pesar de estos resultados, la relación existente entre la satisfacción de las NPB y la IE percibida sigue siendo poco investigada entre los adolescentes (Fernández-Berrocal \& Ramos, 1999; Fernández-Espínola \& Almagro, 2019).

\section{CONCLUSIONES}

En cuanto a las NPB, la mayoría de los estudiantes afirmaron que la NPB que sienten tener más satisfecha fue la relación con los demás, y con respecto a la IE, que es la dimensión reparación emocional en la que puntúan más alto. Finalmente, se demostró que la necesidad psicológica que mayor capacidad de predicción tuvo sobre las dimensiones de la IE fue la relación con los demás, seguida de la percepción de competencia.

\section{REFERENCIAS BIBLIOGRÁFICAS}

Baena-Extremera, A., Gómez-López, M., Granero-Gallegos, A., \& Martínez-Molina, M. (2016). Modelo de predicción de la satisfacción y diversión en Educación Física a partir de la autonomía y el clima motivacional. Universitas Psychologica, 15(2), 39-49. doi:10.11144/Javeriana.upsy15-2.mpsd

Balaguer, I., Castillo, I., \& Duda J.I. (2008). Apoyo a la autonomía, satisfacción de las necesidades, motivación y bienestar en deportistas de competición: Un análisis de la teoría de la autodeterminación. Revista de Psicología del Deporte, 17(1), 123- 139.

Barkoukis, V., Hagger, M.S., Labropoulos, G., \& Tsorbatzoudis, H. (2010). Extending then trans contextual model 
in physical education and leisure time contexts: examining the role of basic psychological need satisfaction. British Journal of Educational Psychology, 80, 647-670. doi:10.1348/000709910X487023

Bar-On, R. (2002). Bar-On Emotional Quotient Inventory: Technical Manual. North Tonawanda, NY.: Multi Health Systems, Inc.

Callea, A., De Rosa, D., Ferri, G., Lipari, F., \& Costanzi, M. (2019). Are more intelligent people happier? emotional intelligence as mediator between need for relatedness, happiness and flourishing. Sustainability, 11, 1022. doi:10.3390/su11041022

Cava, M.J., \& Musitu, G. (2000). Perfil de los niños con problemas de integración social en el aula. Revista de Psicología Social, 15(3), 319-333.

Cera, E., Almagro, B.J., Conde, C., \& Sáenz-López, P. (2015). Inteligencia emocional y motivación en educación física en secundaria. Retos, 27, 8-13.

Deci, E.L, \& Ryan, R.M. (2000). The "what" and "why" of goal pursuits: Human needs and the self determination of behaviour. Psychological Inquiry, 11, 227268.

Deci, E.L., \& Ryan, R.M. (1985). Intrinsic motivation and self determination in human behaviour. New York: Plenum.

Deci, E.L., \& Ryan, R.M. (1991). A motivational approach to self: Integration in personality. En R. Dienstbier (Ed.), Nebraska symposium on motivation: Perspectives on motivation (Vol. 38., pp. 237 288). Lincoln, NE: University of Nebraska Press.

Duncan, S.C. (1993). The role of cognitive appraisal and friendship provisions in adolescents' affect and motivation toward activity in physical education. Research Quarterly for Exercise and Sport, 64(3), 314-323.

Fernández-Berrocal, P., \& Ramos, N. (1999). Investigaciones empíricas en el ámbito de la inteligencia emocional. Ansiedad y Estrés, 5(2-3), 247-260.

Fernández-Berrocal, P., Extremera, N., \& Ramos, N. (2004). Validity and reliability of the Spanish modified version of the trait meta-mood scale. Psychological Reports, 94, 751-755. doi:10.2466/pr0.94.3.751-755

Fernández-Espínola, C., \& Almagro, B.J. (2019). Relación entre motivación e inteligencia emocional en Educación Física: una revisión sistemática. Retos, 36, 584-589.

Fernández-Ozcortaa, E.J., Almagro, B.J., \& Sáenz-López, P. (2014). Explanatory model of psychological wellbeing in the university athletic context. Procedia - Social and Behavioral Sciences, 132, 255-261. doi:10.1016/j.sbspro.2014.04.307

Fierro-Suero, S., Almagro, B.J., \& Sáenz López, P. (2019). Necesidades psicológicas, motivación e inteligencia emocional en Educación Física. Revista Electrónica Interuniversitaria de Formación del Profesorado, 22(2), 167 186. doi:10.6018/reifop.22.2.345241

Franco, E., \& Coterón, J. (2017). The effects of a physical education intervention to support the satisfaction of basic psychological needs on the motivation and intentions to be physically active. Journal of Human Kinetics, 59, 5-15. doi:10.1515/hukin-2017-0143

García-González, L., Aibar, A., Sevil, J., Almolda, F.J., \& Clemente, J.A. (2015). Soporte de autonomía en Educación Física: evidencias para mejorar el proceso de enseñanza. Cultura, Ciencia y Deporte, 10(29), 10311. doi:10.12800/ccd.v10i29.547

Gil, J.A. (2003). Métodos de investigación en educación. Análisis multivariante (vol. III). Madrid: UNED.

Goleman, D. (1995). Emotional Intelligence. New York: Bantam Books.

González-Serrano, G., Huéscar, E., \& Moreno-Murcia, J.A. (2013). Satisfacción con la vida y ejercicio físico. Motricidad. European Journal of Human Movement, 30, 131-151.

Hair, J.F., Black, W.C., Babin, B.J., \& Anderson, R.E: (2009). Multivariate data analysis (7 $7^{\text {th }}$ ed.). New York: Pearson Prentice Hall.

Martín, M., \& Guzmán, J.F. (2012). Inteligencia emocional, motivación autodeterminada y satisfacción de necesidades básicas en el deporte. Cuadernos de Psicología del Deporte, 12(Supl. 2), 39-44. 
Martínez Rubio, R., Granero-Gallegos, A., \& Gómez-López, M. (2020). La satisfacción de las necesidades psicológicas básicas en las clases de Educación Física y su relación con la satisfacción con la vida en los adolescentes. Revista Complutense de Educación, 31(1), 45-53. doi:10.5209/rced.61750

Mayer, J.D., \& Salovey, P. (1990). Emotional intelligence. Imagination, Cognition, and Personality, 9(3), 185211.

Mayer, J.D., \& Salovey, P. (1997). What is emotional intelligence? En P. Salovey \& D.J. Sluyter (Eds.), Emotional development and emotional intelligence: Educational implications (pp. 3-34). New York: Harper Collins.

Mayer, J.D., Salovey, P., \& Caruso, D. (2000). Models of emotional intelligence. En R.J. Sternberg (Ed.), Handbook of Intelligence ( $2^{\text {nd }}$ ed.) (pp. 396-420). New York: Cambridge.

McDonough, M.H., \& Crocker, P.R. (2007). Testing self determined motivation as a mediator of the relationship between psychological needs and affective and behavioral outcomes. Journal of Sport \& Exercise Psychology, 29(5), 645-663. doi:10.1123/jsep.29.5.645

Méndez Giménez, A., Fernández Río, J., \& Cecchini Estrada J. A. (2013). Climas motivacionales, necesidades, motivación y resultados en Educación Física. Aula Abierta, 41, 6372.

Méndez-Giménez, A., Cecchini, J.A., \& García-Romero, C. (2018). Metas de logro 3×2, inteligencia emocional y relaciones sociales en el contexto de la Educación Física. Revista Iberoamericana de Diagnóstico y Evaluación, 49(4), 131-141. doi:10.21865/RIDEP49.4.11

Menéndez, J.I., \& Fernández-Río, J. (2018). Versión española de la escala de necesidades psicológicas básicas en educación física. Revista Internacional de Medicina y Ciencias de la Actividad Física y el Deporte, 18(69), 119-133. doi:10.15366/rimcafd2018.69.008

Montero, I., \& León, 0. (2007). A guide for naming research studies in Psychology. International Journal of Clinical and Health Psychology, 7(3), 847-862.

Moreno, J.A., \& Vera, J. (2011). Modelo causal de la satisfacción con la vida en adolescentes de educación física. Revista de Psicodidáctica, 16(2), 367-380

Moreno, J.A., Hernández, A., \& González-Cutre, D. (2009). Complementando la teoría de la autodeterminación con las metas sociales: un estudio sobre la diversión en educación física. Revista Mexicana de Psicología, 26(2), 213-222.

Moreno-Murcia, J.A., González-Cutre, D., Chillón, M., \& Parra, N. (2008). Adaptación a la educación física de la escala de necesidades psicológicas básicas en el ejercicio. Revista Mexicana de Psicología, 25(2), 298-303.

Ntoumanis, N. (2001). A self-determination approach to the understanding of motivation in physical education. British Journal of Educational Psychology, 71, 225-242. doi:10.1348/000709901158497

Pena, M., Rey, L., \& Extremera, N. (2012). Life satisfaction and engagement in elementary and primary educators: Differences in emotional intelligence and gender. Revista de Psicodidáctica, 17(2), 341-358. doi:10.1387/Rev.Psicodidact.1220

Reeve, J., Jang, H., Carrell, D., Jeon, S., \& Barch, J. (2004). Enhancing students' engagement by increasing teachers' autonomy support. Motivation and Emotion, 28(2), 147-169. doi:10.1023/B:MOEM.0000032312.95499.6f

Reis, H.T., Sheldon, K.M., Gable, S.L., Roscoe, J., \& Ryan, R.M. (2000). Dayly well-being: The role of autonomy, competence, and relatedness. Personality and Social Psychology Bulletin, 26, 419-435.

Ryan, R., \& Deci, E.L. (2017). Self Determination Theory. Basic Psychological Needs in Motivation, Development, and Wellness. New York, NY.: Guilford Press.

Ryan, R.M., \& Deci, E.L. (2000). Self determination theory and the facilitation of intrinsic motivation, social development, and well being. American Psychologist, 55, 6878.

Saarni, C. (1999). The Development of Emotional Competence. New York, NY.: Guilford Press.

Salovey, P., Mayer, J.D., Goldman, S.L., Turvey, C., \& Palfai, T.P. (1995). Emotional attention, clarity, and repair: Exploring emotional intelligence using the Trait Meta-Mood Scale. En J.W. Pennebaker (Ed), Emotion, Disclosure, \& Health (pp. 125-151). Washington: American Psychological Association. 
Vlachopoulos, S.P., \& Michailidou, S. (2006). Development and initial validation of a measure of autonomy, competence, and relatedness in exercise: The Basic Psychological Needs in Exercise Scale. Measurement in Physical Education and Exercise Science, 10, 179-201. doi:10.1207/s15327841mpee1003_4

Vlachopoulos, S.P., Katartzi, E.S., \& Kontou, M.G. (2011). The Basic Psychological Needs in Physical Education Scale. Journal of Teaching in Physical Education, 30, 263-280. doi:10.1080/1091367X.2013.872106

Watson, M., \& Kleinert, J. (2018). The relationship between coaches' emotional intelligence and basic need satisfaction in athletes. Sports Coaching Review. doi:10.1080/21640629.2018.1491669 
